\title{
Percepção de esforço e dispneia em pediatria: revisão das escalas de avaliação
}

\section{Perceived exertion and dyspnea in pediatrics: a review of rating scales}

\author{
Renata Martins ${ }^{1}$, Maíra S. de Assumpção², Camila I. S. Schivinski ${ }^{3}$
}

\begin{abstract}
RESUMO
Modelo do estudo: revisão bibliográfica. Objetivo: revisar e apresentar diferentes escalas utilizadas para avaliação da percepção de esforço e dispneia em crianças e adolescentes. Metodologia: revisão bibliográfica com consulta nas bases de dados: Medline/PubMed e SPORTDiscus (EBSCO), sem restrição de data, realizada no período de fevereiro a maio de 2013. Foram utilizados os descritores: child, perceived exertion, dyspnea, scale. Consideradas publicações que validaram e utilizaram escalas de percepção de esforço e/ou dispneia específicas para crianças e adolescentes. Resultados: foram encontrados 154 estudos, dos quais 12 foram incluídos nessa revisão. Identificou-se um total de 15 escalas validadas na língua inglesa e 1 em português, sendo que a maioria delas utiliza como base a escala de Borg. Cada uma possui particularidades, considerando o tipo de exercício e atividade que será desempenhada, a situação clinica a ser avaliada e idade da criança. Sua adequada aplicação pode retratar informações confiáveis e viabilizar a participação ativa desse grupo etário durante diferentes atividades, auxiliando na área assistencial e cientifica. Conclusões: não há nenhuma escala considerada padrão ouro até o momento. Todas são elaboradas com Figuras, descritores verbais e numéricos, para facilitar a compreensão das crianças e permitir que as mesmas tenham entendimento das próprias sensações de dispneia e de esforço.
\end{abstract}

Palavras-chave: Criança. Métodos de Avaliação. Percepção. Esforço físico. Escalas. Dispneia.

\section{Introdução}

A percepção do esforço (PE) resulta da integração de uma série de informações aferentes oriundas de estruturas sensoriais localizadas nos músculos esqueléticos e no sistema cardiorrespiratório. ${ }^{1}$ A dispnéia é definida como a sensação de desconforto respi- ratório gerado por diversos mecanismos fisiológicos, psíquicos, sociais e ambientais. ${ }^{2}$ Ambas são consideradas indicadores válidos e confiáveis para monitorar e verificar a tolerância do indivíduo ao exercício. ${ }^{3}$

Escalas de avaliação da percepção subjetiva de esforço e dispneia são aplicadas ampla e crescentemente na população adulta ${ }^{4,5}$ e pediátrica ${ }^{6,7}$ No en-
1. Mestranda do Programa de Pós-graduação em Fisioterapia da Universidade do Estado de Santa Catarina, Florianópolis / SC / Brasil. Bolsista FUMDES.

2. Mestranda do Programa de Pós-graduação em Fisioterapia da Universidade do Estado de Santa Catarina, Florianópolis / SC / Brasil. Bolsista CAPES.

3. Docente do Departamento de Fisioterapia da Universidade do Estado de Santa Catarina, Florianópolis / SC / Brasil.
Correspondência Rua Pascoal Simone, 358, Coqueiros CEP: 88080-350. Florianópolis-SC. Laboratório de Fisioterapia Respiratória (LAFIR).

Artigo recebido em 21/08/2013 Aprovado para publicação em 20/12/2013 
tanto, nesse último grupo, o uso eficaz desses instrumentos depende e exige a compreensão da criança, ${ }^{8}$ o que muitas vezes é complexo.

Para tal finalidade, é importante que as abordagens pedagógicas ou estratégias de ensino disponíveis para se aplicar as escalas, proporcionem a capacidade de avançar a compreensão das crianças. Entendo as escalas, as mesmas terão capacidade de conhecer o quanto podem participar de diferentes atividades físicas, bem como o nível de atividade que pode ser apropriado a elas. ${ }^{9}$

Nessa linha, as escalas que vêm sendo desenvolvidas para esse grupo etário, a maioria ao longo dos últimos $20 \operatorname{anos}^{10}$, têm sofrido modificações. São estruturadas com base em 3 diferentes descritores verbais, numéricos, pictóricos - para representar de forma mais clara o esforço/dispneia da criança, e assim facilitar o entendimento do avaliado. ${ }^{7-9,11,12}$

Diante do exposto, este artigo tem por objetivo apresentar algumas das diferentes escalas de percepção de esforço e de dispneia elaboradas e aplicadas para crianças e adolescentes.

\section{Material e Método}

Procedeu-se uma busca na literatura por meio de consultas aos indexadores de pesquisa nas bases de dados Medline/PubMed e SPORTDiscus (EBSCO), sem restrição de idiomas e sem limitação de datas. A pesquisa foi conduzida no período de fevereiro a maio de 2013 e as palavras-chaves utilizadas foram: child, perceveid exertion, dyspnea, scale. Foram consideradas para presente revisão publicações que validaram e que utilizaram pela primeira vez escalas de percepção de esforço e/ou dispneia específicas para crianças e adolescentes.

Uma complementação de material foi feita com base nas referências bibliográficas relacionadas ao tema, sendo elas um resumo ${ }^{13}$ e um artigo clássico. ${ }^{1}$

\section{Resultados}

Inicialmente foram encontrados 154 estudos, e após análise criteriosa de 2 avaliadores independentes, 12 artigos, referentes a estudos de caráter transversal, foram considerados compatíveis com o tema proposto para essa revisão.

Dentre os artigos selecionados nas bases de dados, e os 2 materiais complementares, foram identificadas 16 escalas específicas para faixa etária pe- diátrica ${ }^{1,7-21}$ sendo 15 originais da língua inglesa e uma da língua portuguesa (Brasil) ${ }^{7}$, esta última não validada até o presente momento.

Com base nesses achados, a síntese de dados foi organizada de forma a apresentar as 16 escalas em ordem cronológica.

\section{Escalas de Percepção de Esforço e Dispneia em Pediatria}

\section{Escala de Borg}

A escala de Borg original, descrita em 1974, associa a sensação de esforço durante exercícios físicos com valores referentes à variação da frequência cardíaca (60 a 200 batimentos por minuto) em 15 níveis (6 a 20), sendo " 6 - muito fácil" e " 20 - exaustivo"11 (Figura 1). Esse instrumento é o mais popular da categoria, sendo traduzido para diversas línguas, além da inglesa, e ainda é utilizado mundialmente.

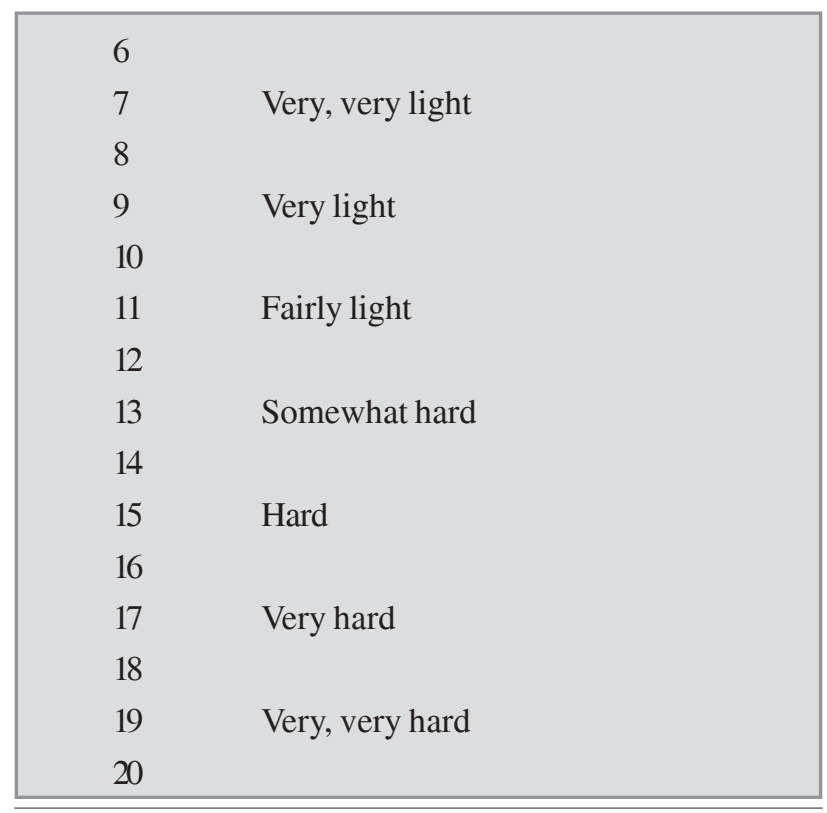

Figura 1: Escala de Borg. (Fonte: Borg (1982).

\section{Escala de Borg Modificada}

Desenvolvida em 1982 para avaliar o grau de percepção do esforço e dispnéia durante o exercício, a escala de Borg Modificada ${ }^{1}$ é uma adaptação da escala de Borg original. ${ }^{11}$ Foi criada originalmente na língua inglesa, mas sofreu diversas traduções para outras línguas, incluindo o português do Brasil. ${ }^{22}$ É uma escala categórica visual, vertical, na qual consta uma numeração de 0 a 10. Cada número está associado a 
uma descrição textual do grau de dispneia, sendo que sua pontuação aumenta à medida que essa sensação também aumenta, de acordo com a descrição numérica referida pelo indivíduo (Figura 2). Essa escala ainda é bastante utilizada na população adulta ${ }^{5,22,23} \mathrm{e}$, apesar da dificuldade em sua compreensão, é frequentemente utilizada em estudos na pediatria. ${ }^{24-29}$

\begin{tabular}{|rl|}
\hline 0 & Nenhuma \\
0,5 & Muito, muito leve \\
1 & Muito leve \\
2 & Leve \\
3 & Moderada \\
4 & Pouca intensa \\
5 & Intensa \\
6 & \\
7 & Muito intensa \\
8 & \\
9 & Muito, muito intensa \\
10 & Máxima \\
\hline \hline
\end{tabular}

Figura 2: Escala de Borg modificada. (Fonte: Cavalcante et al. (2008).

\section{Children's Effort Rating Table (CERT)}

A tabela infantil de avaliação do esforço (CERT) foi criada em 1994, na língua inglesa, através de um processo de interação direta e questionamento de uma amostra de crianças antes, durante, e após participarem de um programa de exercícios. Tem o intuito de avaliar o esforço percebido durante a prática de exercício físico, de diferentes intensidades, em crianças com idade entre 6 e 9 anos. ${ }^{8}$ Envolve valores numéricos de 1 a 10, associados a descritores verbais, sendo "1 - muito, muito fácil" e "10 tão difícil que eu vou parar”, como mostra a Figura 3.

\section{Children's OMNI Scale of Perceived Exertion}

A escala OMNI foi desenvolvida por Robertson et al. $(2000)^{14}$ com o intuito de investigar a medida da percepção do esforço, principalmente, em crianças e adolescentes menores de 11 anos de idade, por apresentarem dificuldade em descrever suas sensações por meio de palavras, ou até mesmo de interpretarem descritores verbais. A escala tem como formato uma faixa resposta numérica de 0 a 10, acompanhada com descritores contínuos, em ordem crescente de intensidades de esforço. Seus descritores pictóricos ilustram uma criança experimentando diversos níveis de esforço ao pedalar uma bicicleta, subindo uma inclinação linear, atividade esta comum na infância. Cada Figura é associada a uma expressão verbal, conforme Figura 4.

\section{Cart and Load Effort Rating (CALER)}

A escala CALER, desenvolvida na língua inglesa por Eston et al. (2000) ${ }^{15}$, retrata uma criança andando de bicicleta em uma superfície horizontal plana, puxando um carrinho. Suas Figuras representam diferentes fases de esforço, caracterizadas pelo aumento progressivo da carga presente no carrinho. Além das Figuras, é composta por descritores verbais e numéricos que vão de " 1 - muito fácil" a " 10 - tão difícil

\begin{tabular}{|rl|}
\hline 1 & Very, Very Easy \\
2 & Very Easy \\
3 & Easy \\
4 & Just Feeling a Strain \\
5 & Starting to Get Hard \\
6 & Getting Quite Hard \\
7 & Hard \\
8 & Very Hard \\
9 & Very, Very Hard \\
10 & So Hard I am Going to Stop \\
\hline \hline
\end{tabular}

Figura 3: Children's Effont Rating Table (CERT). (Fonte: Williams et al. (1994).

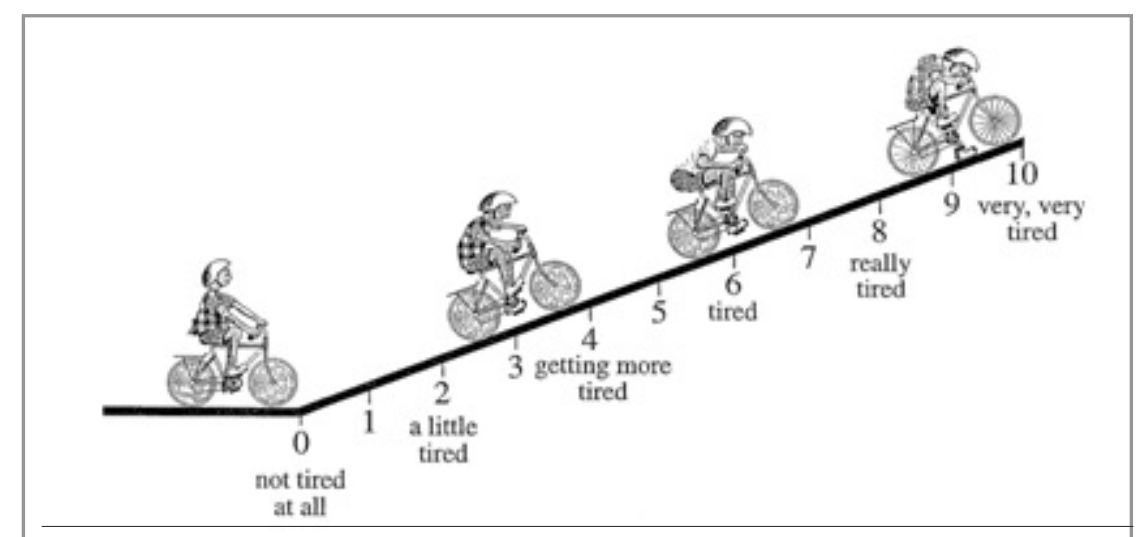

Figura 4: Children's OMNI Scale of Perceived Exertion. (Fonte: Robertson et al. (2000) 
que eu vou parar" 30 (Figura 5). A validade concorrente dessa escala foi avaliada através de diferentes intensidades, em dias separados, mostrando ter associação positiva e significativa com a frequência cardíaca, parâmetro fisiológico de resposta ao esforço ${ }^{31}$.

\section{Bug and Bag Effort Rating Scale (BABE)}

Escala desenvolvida por Eston et al. $(2001)^{16}$, validada na língua inglesa e indicada para crianças com idade entre 7 e 10 anos. É representada pela Figura de um personagem de desenho popular da Disney, uma formiga, que passa por progressivos episódios de esforço físico enquanto pisa sobre um banco carregando uma mochila. O peso da mochila vai aumentando à medida que é preenchida com pedras, conforme Figura 6. O número de pedras na mochila é compatível com números na escala linear, que vai de 0 a 10 , acompanhados de descritores verbais ${ }^{30}$, os mesmos da escala CALER (ESTON et al., 2000). ${ }^{15}$

\section{Scale rating the perceived exertion of young children (RPE-C)}

A RPE-C foi desenvolvida e validada por Groslambert et al. (2001) ${ }^{10}$, para avaliar a percepção de esforço em crianças que não sabem ler (5-6 anos). Essa escala é uma modificação da escala de Borg original, com os mesmos descritores numéricos de 620 , e com os descritores verbais substituidos por imagens coloridas. São 7 Figuras de um homem que torna-se progressivamente cansado, manifestando diferentes expressões faciais para demonstrar diferentes níveis de intensidade do exercício ${ }^{32}$, como apresenta a Figura 7.

\section{Pictorial Children's Effort Rating Table (PCERT)}

Tabela ilustrativa infantil de avaliação do esforço (PCERT), foi desenvolvida em 2002 originalmente em inglês, por Yelling et al. ${ }^{9}$ Segue os mesmos descritores numéricos e verbais da CERT ${ }^{8}$. Seu diferencial está na associação de desenhos de um menino se exercitando, o qual muda de expressão facial e de postura, à medida que o esforço aumenta com a progressão do exercício físico. Classificações mais elevadas correspondem ao aumento na intensidade do exercício (Figura 8 ).

\section{Children's OMNI-walk/run Scale of Perceived Exertion}

A versão ilustrativa da escala OMNI para caminhada e corrida foi criada por Utter et al. em $2002^{17}$, na versão inglesa, para avaliar o esforço percebido pelas crianças nessas atividades. É representada por

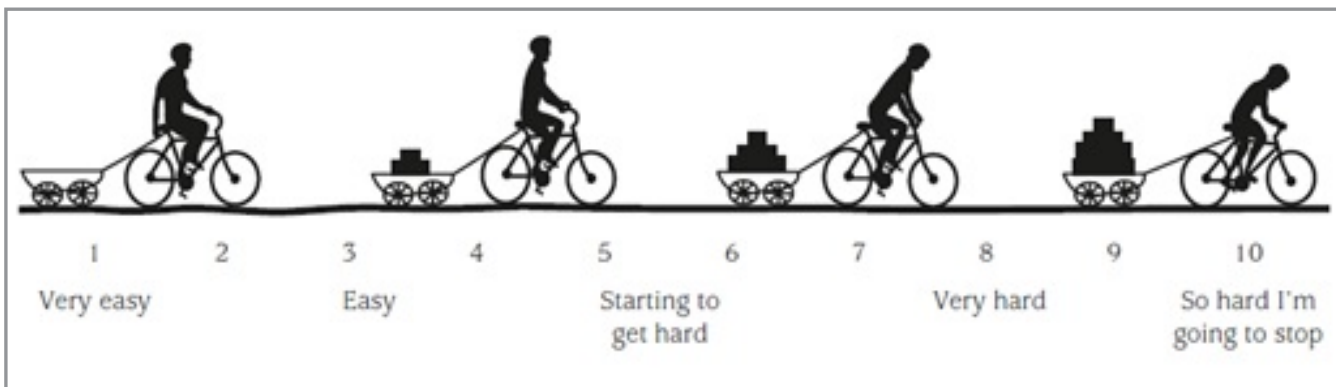

Figura 5: Cart and Load Effort Rating (CALER). (Fonte: Parfitt et al. (2007).

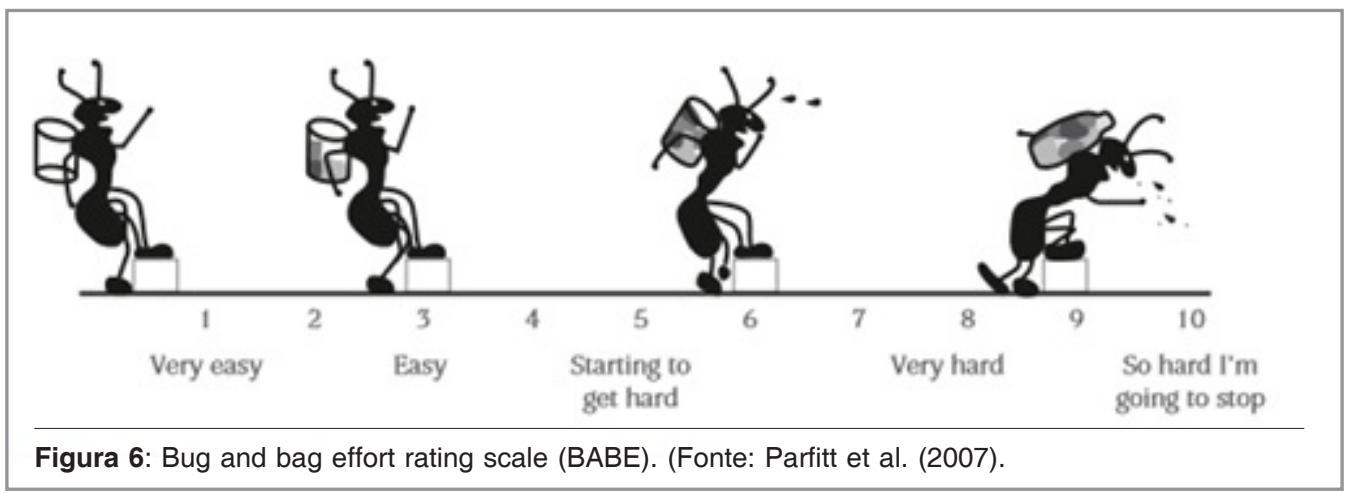


20

19

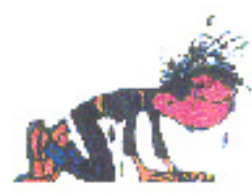

18

17

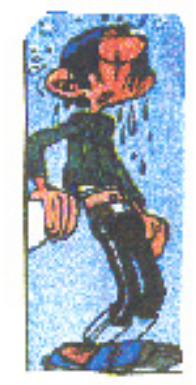

16

15

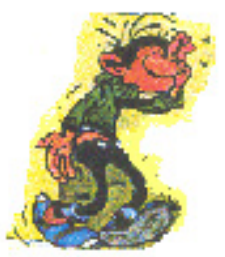

14

13

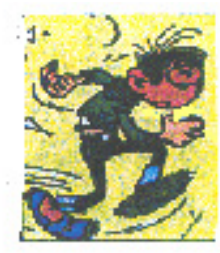

12

11

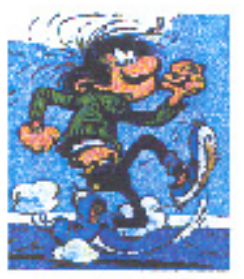

10

9

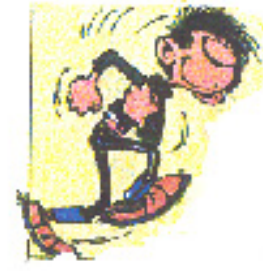

8

7

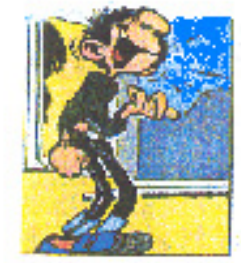

6
3 componentes descritores: verbais, ilustrativos e numéricos. Esses descritores não foram validados individualmente, e sim como um formato único, diferentemente da PCERT. Apresentada na Figura 9, essa escala inclui níveis de esforço pontuados de 0 a 10 , que quantificam os termos de "nem um pouco cansado" até "muito, muito cansado", juntamente com a representação dos desenhos de uma criança ficando cada vez mais cansada a medida que progride seu esforço físico empenhado para subida ao topo de uma colina.

\section{Perceived Exertion Scale for Children (PES-C)}

A escala de percepção de esforço para criança $(P E S-C)$ é um instrumento australiano, validado em 2003 e encontrado somente na língua inglesa. Teve a sua validade estudada em crianças saudáveis com idade de 6 e 7 anos. Além de descritores verbais, apresenta seis ilustrações descritivas, posicionadas ao longo da escala, as quais se atribuem pontuações que

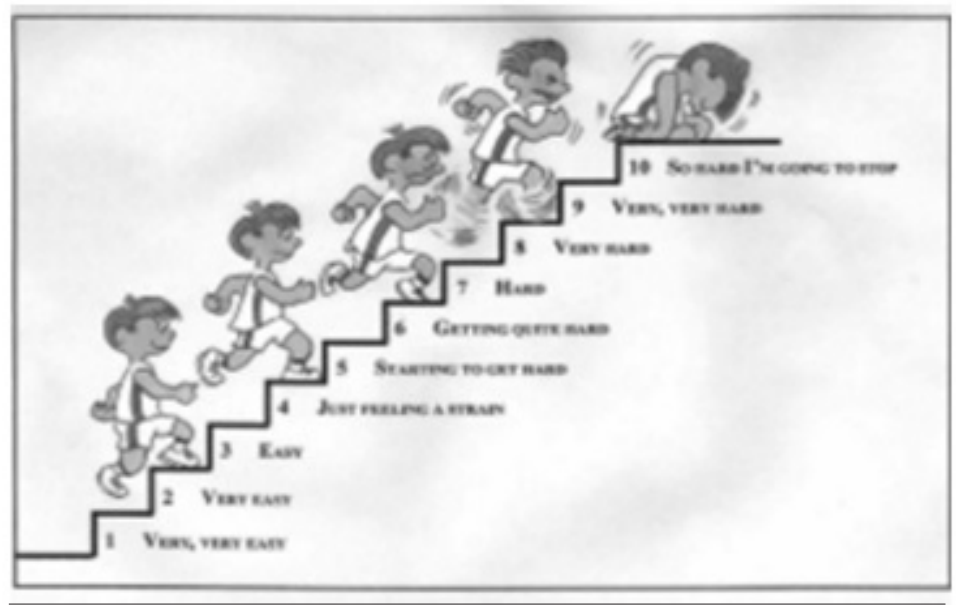

Figura 8: Pctorial Children's Effort Rating Table (PCERT) Fonte: Yelling et al. (2002)

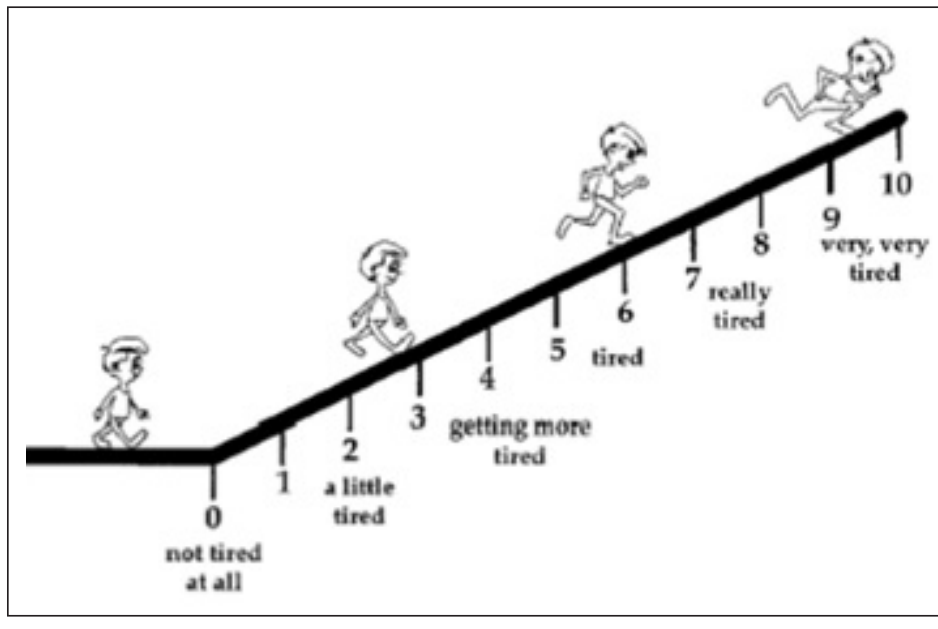

Figura 9: Children's OMNI walk/run scale Fonte: Utter et al. (2001)
Figura 7: Scale rating the perceived exertion of young children (RPE-C) Fonte: Groslambert et al. (2001). 
vão de "0 - Nem um pouco cansado" a "5 - Totalmente exausto. Não posso continuar com o exercício". As ilustrações são constituídas por um urso, cuja postura e expressão facial se modifica à medida que a intensidade do exercício é alterada, sendo que a pontuação da escala aumenta quanto maior for o esforço despendido para a atividade ou exercício ${ }^{13}$ (Figura 10).

\section{Children's OMNI RPE Scale for Stepping Exercise}

Desenvolvida por Robertson et al., em 2005 ${ }^{18}$, a OMNI RPE foi criada na língua inglesa, para avaliar a percepção de esforço em crianças com idade entre 8 e 12 anos, durante um protocolo incremental de subida de degrau. É composta por descritores numéricos que variam de 0 a 10 , descritores verbais (as mesmas escritas da escala OMNI Scale of Perceived Exertion $)^{14}$ e imagens que representam uma criança (menina ou menino) realizando o exercício de subir degraus em uma escada com vários níveis de intensidade, como apresentadas na Figura 11.

\section{Children's OMNI Resistance Exercise Scale (OMNI-RES)}

\section{A OMNI-res}

teve como primeiro objetivo avaliar o esforço percebido por crianças e adolescentes, de 10 a 14 anos de idade, durante a realização de exercícios isotônicos de membros supe-

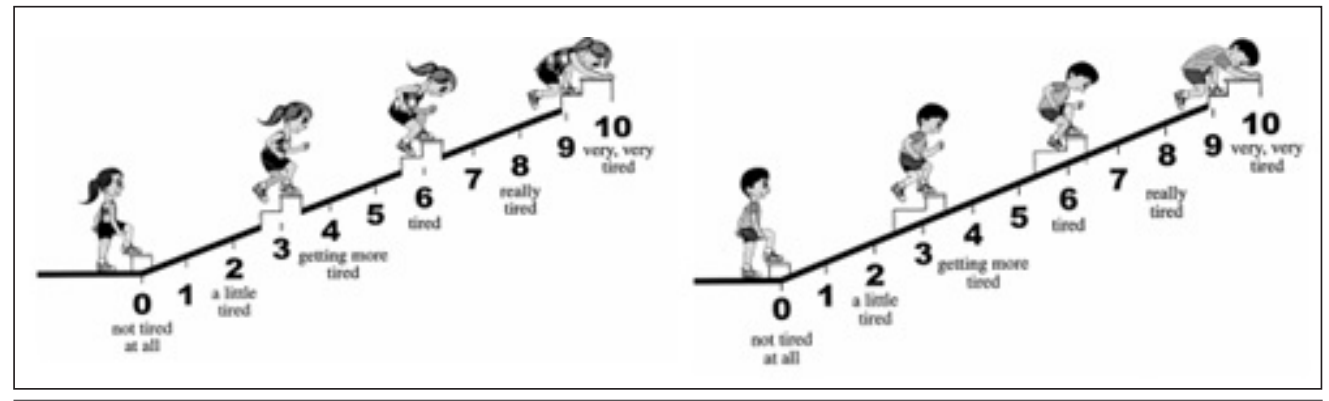

Figura 11: Children's OMNIRPE scale for stepping exercice. Fonte: Robertson et al. (2005-a).

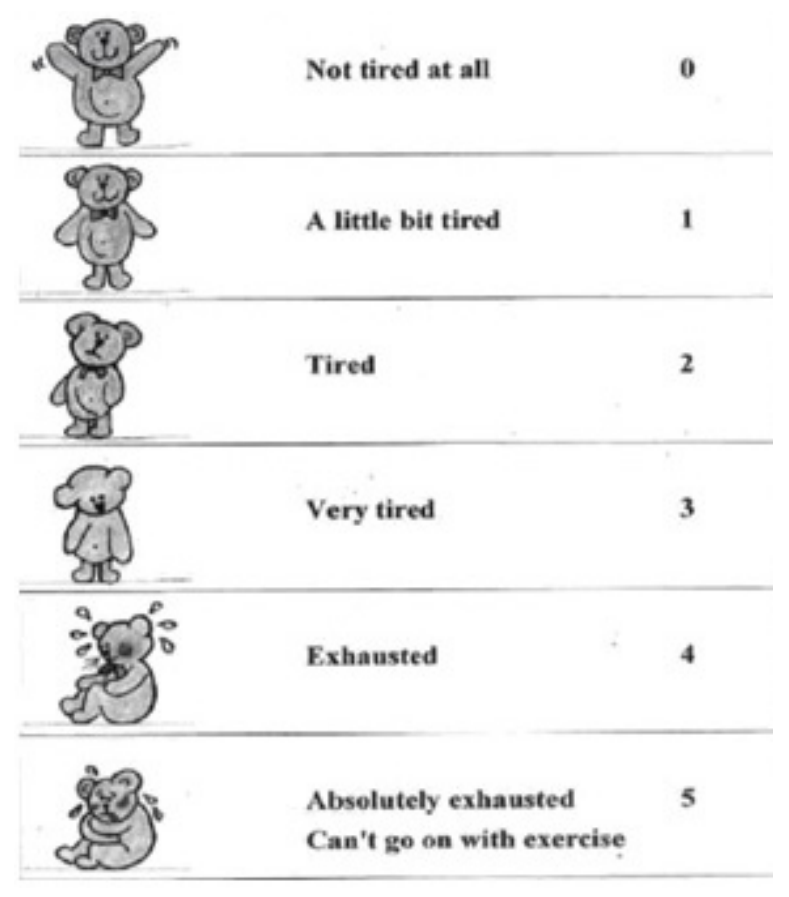

Figura 10: Perceived Exertion Scale for Children (PES-C) Fonte: Simon et al. (2003) riores e inferiores. Criada por Robertson et al., em $2005^{19}$, a escala apresenta descritores verbais, numéricos e pictóricos, e é representada por uma criança levantando peso com os membros superiores. Sua pontuação varia de " 0 - extremamente fácil" a "10 - extremamente difícil" (Figura 12).

\section{Dalhousie Dyspnea Scales}

Com o objetivo de desenvolver uma escala pictórica que abrangesse a percepção de dispnéia em crianças acima de 8 anos de idade, em 2005

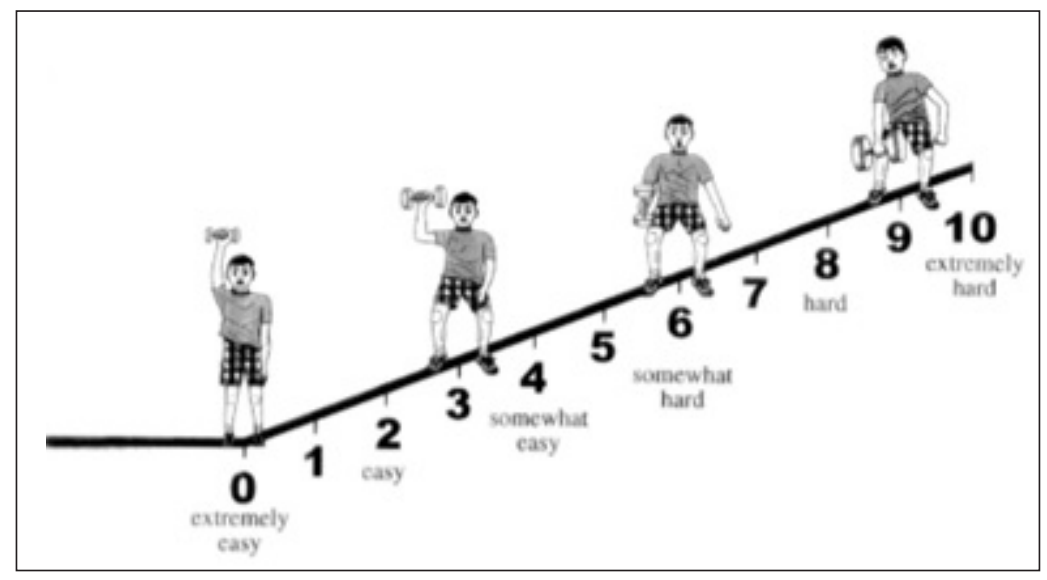

Figura 12: Children's OMNI resistance exercise scale (OMNI-RES). Fonte: Robertson et al. (2005-b). 
McGraft e colaboradores ${ }^{20}$ criaram a escala de dispneia Dalhousie. Com propriedades ordinais, foi elaborada para não ter uma magnitude tão ampla como a escala de Borg. É constituída por imagens que descrevem o auto-relato da dispneia de crianças e adolescentes através de uma combinação de três fatores: "fechamento da garganta", "aperto no peito" e esforço. Cada um desses elementos é representado por escalas com 7 imagens individuais, crescentes em gravidade de esforço, através de cartões laminados de $12 \times 15$ centímetros. As três escalas possuem "âncoras" com pontuações de 0 (nenhum) a 10 (máximo) de dispneia (fechamento da garganta, sensação de aperto no peito e esforço (Figura 13).

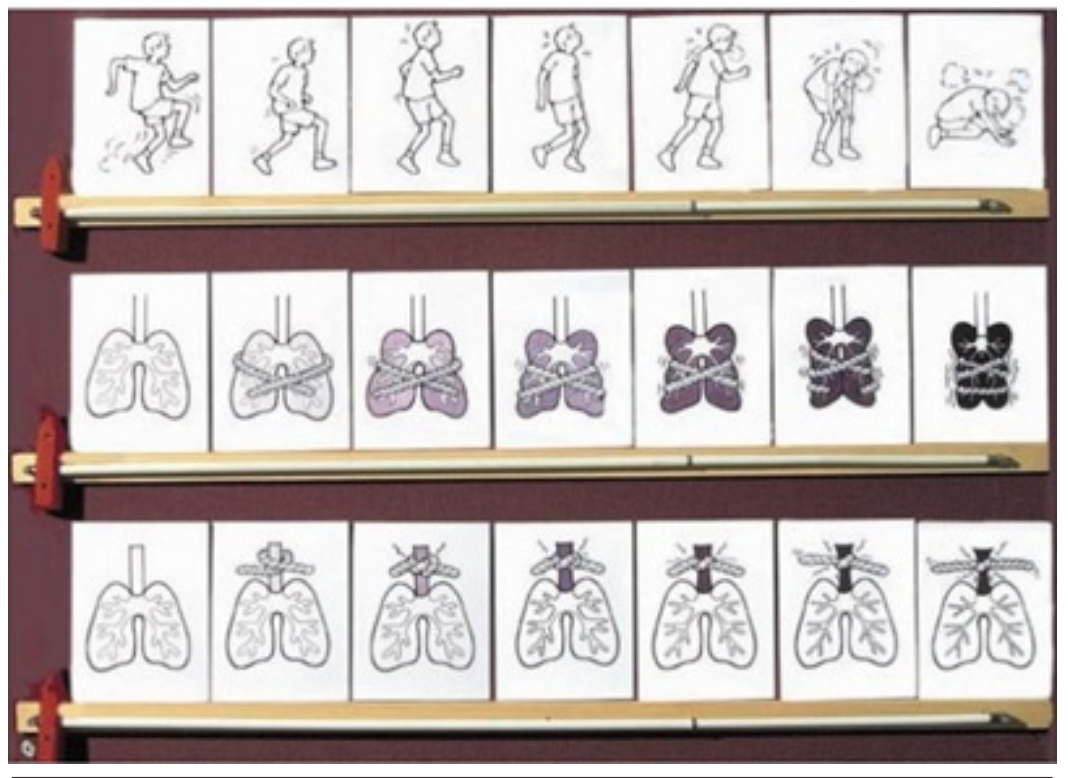

Figura 13: Dalhousie Dyspnea Scales. Fonte: McGrath et al. (2005).

\section{Pediatric Dyspnea Scale}

A escala pediátrica de dispneia foi desenvolvida em 2009 por Khan et al. ${ }^{21}$, com o objetivo de apoiar a tomada de decisão no manejo de pacientes asmáticos hospitalizados, e comparar o desempenho da escala com marcadores tradicionais de monitoramento da asma na previsão de resultados após a alta dos pacientes. É uma escala de classificação subjetiva dos sintomas em relação à dificuldade apresentada para respirar. É composta por 3 itens ilustrativos: uma série de expressões faciais para estimar a sensação geral de bem-estar ou desconforto (baseado na escala de Wong-Baker ${ }^{33}$; Figuras do pulmão para avaliar a sensação de aperto no peito (baseada na escala de Dalhousie) $)^{20}$; e uma escala de cores com descrições verbais para representar a intensidade da dificuldade em respirar. A pontuação varia de 1 a 7, no qual o " 1 - sem dificuldade alguma" e "7 - muitíssima dificuldade" de respirar (Figura 14).

\section{Pictorial curvilinear ratings of perceived exertion scale}

A escala de percepção de esforço com classificações pictóricas curvilíneas foi validada para crianças saudáveis de 7 a 8 anos em 2009, por Eston et al. ${ }^{12}$ Foi desenvolvida com o objetivo de verificar se a percepção de esforço acompanha de maneira curvilínea à imposição de um ritmo de trabalho com o aumento na intensidade do exercício. No estudo de validação esse

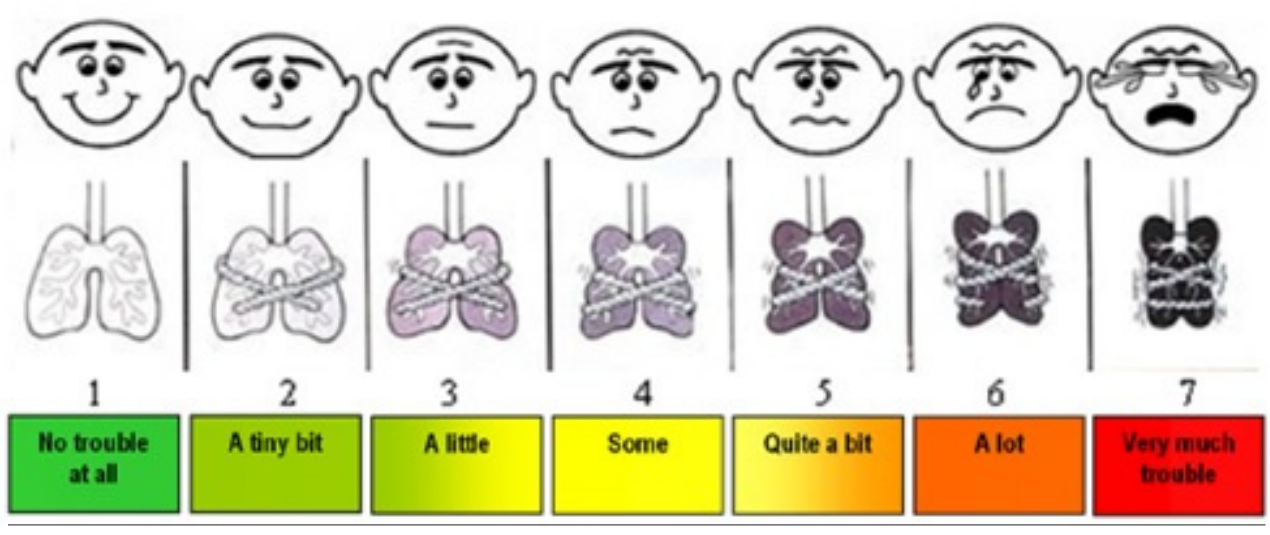

Figura 14: Pediatric Dyspnea Scale. Fonte: Khan et al. (2009). 
exercício foi realizado com uma bicicleta ergométrica. A escala utiliza 5 descritores verbais, o mesmo intervalo numérico da CERT, e imagens que retratam um personagem em vários estágios de esforço. Essas imagens estão dispostas em uma inclinação de forma côncava, com aumento progressivo do gradiente de intensidade. A distância entre cada incremento é numerada no eixo horizontal $(0-10)$ e ha a representação de uma curva sombreada de maneira progressiva, de cor clara ao escuro, da esquerda para a direita, como mostra a Figura 15.

\section{Escala Visual Analógica (EVA) Legendada}

A escala visual analógica legendada de dispneia (EVA) foi criada primeiramente na língua portuguesa (Brasil) e utilizada por Lima et al. $(2010)^{7}$ para determinar o grau de dispneia durante teste de broncoespasmo induzido por exercício em crianças e adolescentes asmáticos. Representada na Figura 16, apresenta escore de 0 a 3 , associado a uma sequência de ilustrações da performance de um garoto fazendo exercícios com uma bola. Uma extremidade da escala sinaliza ausência de sintomas ( 0 pontos) e a outra simboliza dispneia grave (3 pontos).

\section{Discussão}

Para avaliação da percepção de esforço e dispnéia em crianças e adolescentes, especialmente em menores de 10 anos, dispositivos desenvolvidos para chamar a atenção e facilitar a compreensão são necessários para derivar informações válidas e confiáveis sobre diferentes atividades, bem como orientações bem detalhadas são fundamentais para sua aplicação ${ }^{8}$. Esses dispositivos são instrumentos mundialmente utilizados, tanto na população adulta quanto pediátrica, as escalas, indicadas em várias situações, como testes de esforço ${ }^{3-5}$, prescrições de exercícios ${ }^{12,15,19,27}$ e em situações clínicas ${ }^{21,22,28}$. (2009).

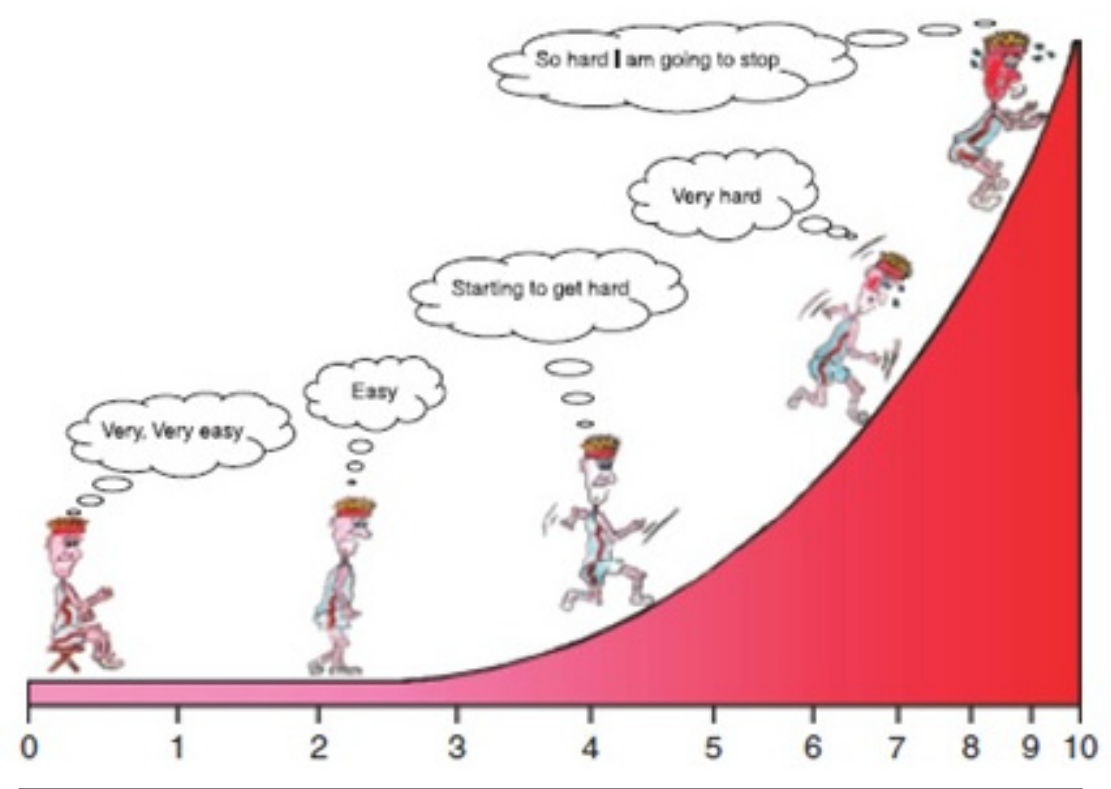

Figura 15: Pictorial curvilinear ratings of perceived exertion scale. Fonte: Eston et al.

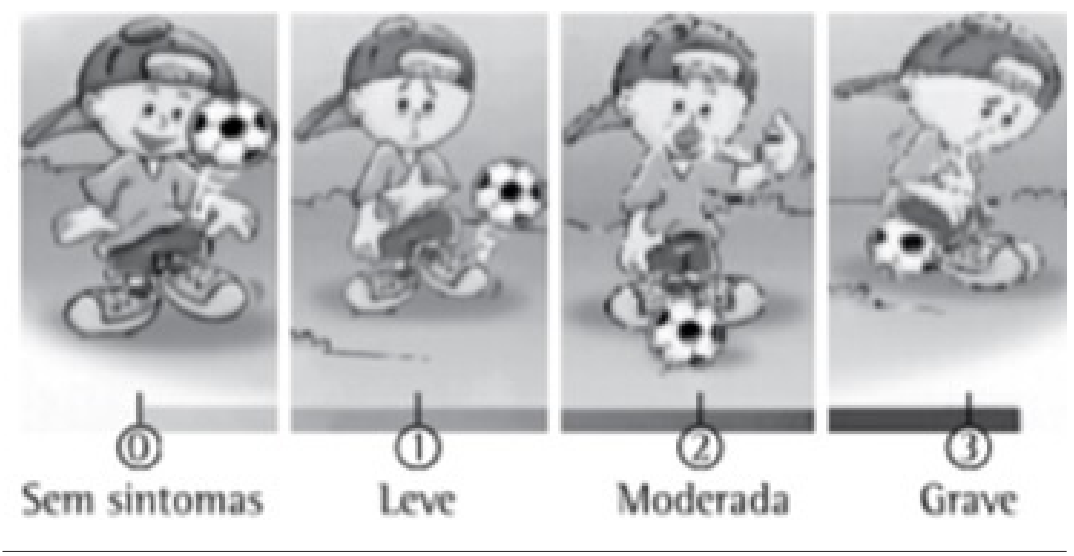

Figura 16: Escala visual analógica legendada de dispnéia. Fonte: Lima et al. (2010).

$\mathrm{Na}$ presente revisão, foram identificadas 16 escalas específicas para avaliação da percepção de esforço e dispnéia em pediatria, sendo todas muito semelhantes no que diz respeito à estrutura, uma vez que apresentam ilustrações, descritores numéricos e verbais. Treze destes instrumentos possuem Figuras ${ }^{7}$, 9, 10,12-21 e apenas dois não utilizam descritores verbais ${ }^{10,20}$, sendo que o diferencial entre eles é a parte pictórica. No entanto, o que chama a atenção, é o fato da maioria deles utilizar como base a escala de Borg, que apesar de ser extremamente útil clinicamente, uma vez que se correlaciona bem com os parâmetros fisiológicos, utiliza apenas descritores verbais e numéricos (6-20) e exige nível de compreensão elevado, o que torna difícil a sua aplicação em crianças ${ }^{34}$. 
Essa limitação da escala é reforçada no único ensaio clinico utilizando a escala de Borg aqui identificado $^{35}$. Essa pesquisa avaliou a utilidade dessa escala na percepção do esforço de crianças com sobrepeso, idades entre 9 e 15, como um meio para prescrição do exercício. Os participantes pedalaram em quatro intensidades diferentes e referiram a classificação de percepção de esforço segundo a escala. Na sequencia, foram convidadas a definir a resistência no ergômetro e na caminhada/corrida em intensidades que eles percebiam ser igual a 7, 10, 13 e 16. Os autores concluíram que crianças com excesso de peso precisam de instrução adicional na utilização da escala de Borg para a prescrição de exercícios.

Já a escala de Borg Modificada vem sendo frequentemente utilizada em algumas pesquisas ${ }^{24-29}$ como método de avaliação, sendo este um instrumento considerado útil para a mensuração da dispneia após uma determinada tarefa, como um teste de exercício. Dentre elas, Marinov et al. (2008) ${ }^{25}$, compararam a escala Modificada de Borg com a PCERT em um grupo de 50 crianças saudáveis, com média de idade de 10,5 anos, durante um teste incremental em esteira. Os autores concluíram que a PCERT parece ser mais sensível e de mais fácil entendimento, uma vez que as crianças têm uma melhor compreensão do esforço percebido quando uma combinação de descritores pictóricos e verbal é utilizada. Diferentemente, em estudo que comparou a Borg Modificada com a EVA legendada, a primeira foi considerada mais acurada por ter a opção de 10 pontos de classificação, uma vez que a EVA disponibiliza uma restrita faixa de pontuação (4 pontos), o que pode resultar na imprecisão da avaliação do esforço. ${ }^{7}$

Apesar dessa característica da EVA Legendada $(2010)^{7}$, assim como a Pediatric Dyspnea Scale $(2009)^{21}$, a EVA foi criada para populações infantis específicas. Ambas foram desenvolvidas para o controle de crianças com asma, tendo grande relevância aos profissionais da área da saúde no manejo de seus pacientes, no que concerne o monitoramento clinico e a verificação da resposta aos tratamentos realizados. ${ }^{36}$

Nesse âmbito, observa-se que não há uma escala definida como padrão ouro na avaliação da percepção do esforço e/ou dispnéia em crianças. Cada uma apresenta suas particularidades e características, que direcionam a escolha e aplicabilidade clínica. Mas dentre os artigos selecionados, verificou-se que os instrumentos mais utilizados nos estudos com a população infantil são a $O M N I^{14,17,18,19,37,38,39}$, PCERT $^{9,25,37}$ e a Borg modificada ${ }^{1,24-29}$, como já mencionado.
As escalas que se apresentam no formato OMNI possuem em comum descritores verbais e visuais simples ${ }^{40}$, com a finalidade de facilitar o entendimento das categorias apresentadas, o que é fundamental para crianças. Para isso, disponibilizam diversos modelos de atividade como, andar/correr, ciclismo, resistência e protocolos de subir degraus.

Na comparação desse tipo de escala com outro formato, Roemmich et al. (2006) $)^{37}$ analisaram a validade das escalas OMNI walk/run e PCERT em 51 crianças com média de idade de 11 anos durante um teste incremental em esteira. Em ambas, o esforço percebido cresceu de forma linear com o aumento da intensidade do exercício, havendo alta correlação $(r=0,92)$ entre os dois instrumentos. Os autores concluíram que, para avaliação do esforço percebido por crianças no exercício submáximo, tanto uma como a outra são aceitáveis.

Nessa linha, verifica-se que os estudos comparativos direcionam para escolha de uma ou outra escala, considerando o tipo de exercício e atividade que será desempenhada, a situação clinica a ser avaliada e idade da criança. Além de nortear para escolha do instrumento mais adequado, tais dados proporcionam maior compreensão das possíveis alterações apresentadas durante a execução de atividades e exercícios, no que se refere à sensação de dispneia e/ou o esforço.

Estudos ressaltam ainda que uma aplicação efetiva deste tipo de instrumento depende da experiência clínica de cada avaliador com a tarefa a ser executada e com o manuseio do instrumento escolhido, além do domínio quanto ao propósito da avaliação em questão e o grau de entendimento da criança a ser avalia$\mathrm{da}^{12,26,41}$, assim como mostra o estudo de Turchetta et al. $2011^{42}$, que avaliou crianças com hérnia diafragmática congênita e utilizou a escala de Dalhousie, que apesar de ter sido desenvolvida para abranger crianças maiores de 8 anos de idade, mostrou ser uma ótima ferramenta de apoio para a pesquisa, mesmo quando aplicada em crianças menores que essa faixa etária.

Diante do exposto na presente revisão, fica evidente que esses instrumentos são importantes ferramentas na prática clínica e no embasamento de pesquisas científicas. Cada qual com suas especificidades, todas as escalas têm como principal objetivo viabilizar o entendimento das crianças quanto às suas sensações de dispneia e/ou esforço, auxiliando no auto-monitoramento do desempenho durante atividades e exercícios e subsidiando examinadores nessa questão. 


\section{Conclusão}

São várias as escalas elaboradas para faixa etária pediátrica, não havendo nenhuma considerada padrão ouro até o momento. Apesar de algumas particularidades, todas são elaboradas com Figuras, descrito- res verbais e numéricos, para facilitar a compreensão das crianças e permitir que as mesmas tenham entendimento das próprias sensações de dispnéia e de esforço. A adequada aplicação desse instrumento pode retratar informações confiáveis e viabilizar a participação ativa desse grupo etário durante diferentes atividades.

\begin{abstract}
Design of the study: literature review. Objective: to review and present different scales used to evaluate the perceived exertion and dyspnea in children and adolescents. Methodology: a literature review to researching in the electronic databases: Medline / PubMed and SPORTDiscus (EBSCO), no date restriction in the period of February to May 2013. Used the following keywords: child, Perceived exertion, dyspnea, scale. Publications that used and validated perceived exertion and / or dyspnea scales specific to children and adolescents were considered. Results: 154 studies were found, of which 12 were included in this review. Were identified a total of 15 validated scales in English and one in Portuguese, with the majority of them to build on the Borg scale. Each has its own peculiarities, considering the type of exercise and activity to be performed, the clinical situation to be assessed and the child's age. Its proper application can portray reliable information and facilitate the active participation of this age group during different activities, assisting in the care and science. Conclusions: there is no scale considered the gold standard so far. All scales are elaborated with figures, verbal and numerical descriptors, to facilitate understanding of the children and allow them to have their own understanding of dyspnea and effort.
\end{abstract}

Keywords: Child. Evaluation Methods. Perception. Physical exertion. Scales. Dyspnea.

\section{Referências bibliográficas}

1. Borg GAV. Psychophysical bases of perceived exertion. Med Sci Sports Exerc. 1982;14:377-81.

2. Of?cial Statement of the ATS. Dyspnea. Mechanisms, assessment, and management: a consensus statement. American Thoracic Society. Am J Respir Crit Care Med. 1999;159: 32140.

3. American College of Sports Medicine. Diretrizes do ACSM para os testes de esforço e sua prescrição. Rio de Janeiro: Guanabara Koogan; 2003.

4. Barros JF, Valle GP, Godoy JRP. Percepção subjetiva do esforço em indivíduos hipertensos e normotensos durante a execução do teste ergométrico. Fisioter Bras. 2004;5:194-201.

5. Mazzocchi CS, Costa CC, Canterle DB, Moussalle LD, Colombo C, Teixeira PJZ. Comparação das variáveis fisiológicas no teste de caminhada de seis minutos e no teste da escada em portadores de doença pulmonar obstrutiva crônica. Rev Bras Med Esporte. 2012;18:296-9.

6. Marinov B, Kostianev S, Turnovska T. Ventilatory efficiency and rate of perceived exertion in obese and non?obese children performing standardized exercise. Clin Physiol Funct Imaging. 2002;22:254-60.

7. Lima PB, Santoro IL, Caetano LB, Cabral ALB, Fernandes ALG. Desempenho de uma escala analógica visual legendada na determinação do grau de dispneia durante teste de broncoespasmo induzido por exercício em crianças e adolescentes asmáticos. J Bras Pneumol. 2010;36:532-8.
8. Williams JG, Eston R, Furlong B. CERT: A perceived exertion scale for young children. Percept Mot Skills. 1994;79:1451-8.

9. Yelling M, Lamb KL, Swaine IL. Validity of a pictorial perceived exertion scale for effort estimation and effort production during stepping exercise in adolescent children. European Physical Education Review 2002;8:157-75.

10. Groslambert A, Hintzy F, Hoffman MD, Dugué B, Rouillon JD. Validation of a rating scale of perceived exertion in young children. Int J Sports Med. 2001;22:116-9.

11. Borg G \& Noble B. Perceived exertion. In: Exercise and Sports Sciences Review, J.H. Wilmore (Ed.). New York: Academic Press, 1974, pp. 131-53.

12. Eston RG, Lambrick DM, Rowlands AV. The perceptual response to exercise of progressively increasing intensity in children aged 7-8 years: Validation of a pictorial curvilinear ratings of perceived exertion scale. Psychophysiology. 2009;46:843-51.

13. Simon S, Alison J, Dwyer G, Follett J. Validation of a perceived exertion scale for young children. [Abstract]. Australian Physiotherapy Association-National Paediatric Conference; 2003.

14. Robertson RJ, Goss FL, Boer NF, Peoples JA, Foreman AJ, Dabayebeh IM, Thompkins, T. Children's OMNI scale of perceived exertion: mixed gender and race validation. Med Sci Sports Exerc. 2000;32:452-8.

15. Eston RG, Parfitt G, Campbell L, Lamb KL. Reliability of effort perception for regulating exercise intensity in children using a Cart and Load Effort Rating (CALER) Scale. Pediatr Exerc Sci 2000;12:388-97. 
16. Eston RG, Parfitt G, Shepherd P. Effort perception in children implications for validity and reliability. In: Papaionnou A, Goudas M, Theodorakis Y, (eds). Proceedings of 10th World Congress of Sport Psychology, Skiathos, Greece. 2001; 5:104-6.

17. Utter AC, Robertson RJ, Nieman DC, Kang J. Children's OMNI Scale of Perceived Exertion: walking/running evaluation. Med Sci Sports Exerc. 2002;34:139-44.

18. Robertson RJ, Goss FL, Andreacci JL, Dube JJ, Rutkowski JJ, Snee BM, Metz K F. Validation of the children's OMNI RPE scale for stepping exercise. Med Sci Sports Exerc. 2005a;37:290-8.

19. Robertson RJ, Goss FL, Andreacci JL, Dube JJ, Rutkowski JJ, Frazee KM, Snee BM. Validation of the Children's OMNIResistance Exercise Scale of perceived exertion. Med Sci Sports Exerc. 2005-b;37:819-26.

20. McGrath PJ, Pianosi PT, Unruh AM, Buckley CP. Dalhousie dyspnea scales: Construct and content validity of pictorial scales for measuring dyspnea. BMC Pediatr 2005;5:33.

21. Khan FI, Reddy RC, Baptist AP. Pediatric Dyspnea Scale for use in hospitalized patients with asthma. J Allergy Clin Immunol. 2009;123:660-4.

22. Cavalcante TMC, Diccini S, Barbosa DA, Bittencourt ARC. Uso da escala modificada de Borg na crise asmática. Acta Paul Enferm. 2008;21:466-73.

23. Pessoa IMS, Costa D, Velloso M, Mancuzo E, Reis MA, Parreira VF. Efeitos da ventilação não-invasiva sobre a hiperinsuflação dinâmica de pacientes com DPOC durante atividade da vida diária com os membros superiores. Rev Bras Fisioter. 2012;16(1):61-7.

24. Cunha MT, Rozov T, de Oliveira RC, Jardim JR. Six-minute walk test in children and adolescents with cystic fibrosis. Pediatr Pulmonol. 2006;41:618-22.

25. Marinov B, Mandadjieva S, Kostianev S. Pictorial and verbal category? ratio scales for effort estimation in children. Child Care Health Dev. 2008;34:35-43.

26. Hommerding PX, Donadio MV, Paim TF, Marostica PJ. The Borg scale is accurate in children and adolescents older than 9 years with cystic fibrosis. Respir Care. 2010;55:72933.

27. Macedo TMF, Oliveira KMC, Melo JBC, Medeiros MG, Medeiros FWC, Ferreira GMH et al. Treinamento muscular inspiratório em crianças com leucemia aguda: resultados preliminares. Rev Paul Pediatr. 2010;28:352-8.

28. Parente AAMI, March MFP, Evangelista LA, Cunha AL. Percepção da dispneia na crise asmática pediátrica pelos pacientes e responsáveis. J Pediatr. 2011;87:541-6.

29. Oliveira JS, Campos TF, Borja RO, Silva ROE, Freitas DA, Oliveira LC, Mendonça KMPP. Analysis of the rate of perceived exertion in the assessment of maximal respiratory pressures in children and adolescents. Rev Bras Crescimento Desenvolv Hum. 2012; 22:314-20.
30. Parfitt G, Shepherd P, Eston RG. Reliability of effort production using the children's CALER and BABE perceived exertion scales. J Exerc Sci Fit. 2007;5:49-55.

31. Barkley JE, Roemmich JN. Validity of a pediatric rpe scale when different exercise intensities are completed on separate days. J Exerc Sci Fit. 2011;9:52-7.

32. Groslambert A, Nachon M, Rouillon JD. Influence of the age on self regulation of static grip forces from perceived exertion values. Neurosci Lett. 2002;325:52-6.

33. Garra G, Singer AJ, Taira BR, Chohan J, Cardoz H, Chisena E, Thode HC. Validation of the Wong-Baker FACES Pain Rating Scale in Pediatric Emergency Department Patients. Acad Emerg Med. 2010;17:50-4.

34. Aquino ES, Mourão FAG, Souza RKV, Glicério BM, Coelho CC Análise comparativa do teste de caminhada de seis minutos em crianças e adolescentes saudáveis. Rev Bras Fisioter. 2010;14:75-80.

35. Ward DS, Bar-Or O. Use of the Borg scale in exercise prescription for overweight youth. Can J Sport Sci. 1990;15:120-5.

36. Cavallazzi TGL, Cavallazzi RS, Cavalvante TMC, Bettencourt ARC, Diccin S. Avaliação do uso da Escala Modificada de Borg na crise asmática. Acta Paul Enferm. 2005;18:39-45.

37. Roemmich JN, Barkley JE, Epstein LH, Lobarinas CL, White TM, Foster JH. Validity of PCERT and OMNI walk/run ratings of perceived exertion. Med Sci Sports Exerc. 2006;38:1014-19.

38. Robertson RJ, Fredric LG, Aaron DJ, Gairola A, Kowallis RA, Liu $Y$ et al. One repetition maximum prediction models for children using the OMNI RPE scale. J Strength Cond Res. 2008;22:196-201.

39. Mahon AD, Woodruff ME, Horn MP, Marjerrison AD, Cole AS. 40. Effect of stimulant medication use by children with ADHD on heart rate and perceived exertion. Adapt Phys Actv $Q$ 2012;29:151-60.

40. Calil e Silva A, Dias MRC, Bara Filho M, Lima JRP, Damasceno VO, Miranda $\mathrm{H}$ et al. Escalas de Borg e OMNI na prescrição de exercício em cicloergômetro. Rev Bras Cineantropom Desempenho Hum. 2011;13:117-23.

41. Groslambert A, Mahon AD. Perceived exertion: influence of age and cognitive development. Sports Med. 2006; 36:911-28.

42. Turchetta A, Fintini D, Ca?ero G, Calzolari A, Giordano U, Cutrera R, et al. Physical Activity, Fitness, and Dyspnea Perception in Children with Congenital Diaphragmatic Hernia. Pediatr Pulmonol. 2011;46:1000-6. 